\title{
Electrical Resistivity of the Monoatomic Metallic Layer
}

\author{
A. PAJA* AND A. DZIAŁO \\ AGH University of Science and Technology, Faculty of Physics and Applied Computer Science \\ Department of Solid State Physics, al. A. Mickiewicza 30, 30-059 Krakow, Poland
}

(Received December 31, 2012; in final form March 8, 2013)

\begin{abstract}
We present new formula which describes the change of electrical resistivity of a monoatomic metallic layer with temperature. The results are compared with those given by the Bloch-Grüneisen formula for bulk metals. Our calculated values compared with those for bulk materials are significantly higher at low temperatures $(T<0.1 \theta)$ and apparently lower at the remaining range of temperatures. Both effects can be explained by the low dimensionality of the sample.
\end{abstract}

DOI: $10.12693 /$ APhysPolA.123.770

PACS: 73.50.-h, 73.50.Bk, 73.63.-b, 73.63.Hs

\section{Introduction}

In recent decades nanotechnology has contributed largely in various fields including electronics, material science, chemistry and biology. There is no doubt that conductivity (or resistivity) of bulk metallic materials should in apparent way differ from that of materials downsized to nanoscale. That is why transport properties of charge and spin in such systems have been the field of interests for many scientists. Fuchs [1] and Sondheimer [2] explained the increase of resistivity with decrease of film thickness in a semiclassical way. In this theory the change of the resistivity, $\rho$, comes from diffuse surface scattering events when the ratio of film thickness to electronic mean free path, $d / l$, becomes small. Later theoretical studies on quantum size effects in thin metallic films with perfect surfaces were made by Sandomirskii [3]. More extended work by Ashcroft and Trivedi [4] involves discreteness of the energy levels, effects of impurity potentials and surface roughness. Palasantzas and De Hosson [5] also investigated the influence of roughness on the electrical conductivity of thin semiconducting and metallic films. They simulated quasi-layer-by-layer growth of the film and described the electron transport by means of Boltzmann-like transport theory. Meyerovich and Ponomarev [6] studied the effect of surface roughness on quantum size effects in thin films. They calculated the dependence of the conductivity on the film thickness. Electronic properties of ultrathin $\mathrm{Cu}$ and Fe films in the thickness range between 1 and 32 monolayers were studied by Fedorov et al. [7]. They got interesting results for the density of states and plasma oscillations but presented no results for conductivity. Experimental results of the resistivity in very thin copper films (approximately 10$150 \mathrm{~nm}$ thick) were obtained by Yarimbiyik et al. [8]. They found the dependence of the resistivity on the thickness but not on the temperature.

*corresponding author; e-mail: paja@agh.edu.pl
The influence of temperature on the resistivity of nanosystems rarely was the subject of interest. To our knowledge only Kravchenko and Klapwijk [9] measured the temperature dependence of the resistivity in a two-dimensional (2D) electron system in the temperature range from $35 \mathrm{mK}$ to $1 \mathrm{~K}$. Thus we took up the task to calculate theoretically this dependence for such system in a wider range of temperatures. The theory describing the dependence of the electrical resistivity of metals on temperature was first introduced by Bloch [10, 11]. However this theory was formulated for bulk materials then it is not immediately applicable to systems strongly constricted in one or more dimensions. Fortunately, the quasiclassical approach based on the Boltzmann equation may be applied to this problem after appropriate modifications [12, 13].

In this article we consider a single mono-atomic metallic layer through which a 2D electron gas is moving. Charge carriers are scattered by thermal lattice vibrations, i.e. phonons. The system is assumed to be exactly two-dimensional and is treated semiclassically. The electrical resistivity calculated on this basis differs from the well-known Bloch-Grüneisen formula.

\section{Theoretical model}

Before we proceed with calculations we are going to say a few words about the model. Because of reduction of the dimensions we are replacing the Fermi/Debye sphere by a Fermi/Debye circle so that every electron/phonon wave vector lies in a plane within the circle. For clarity we do not use different symbols for $k$, $q$, etc. but we shall keep in mind that our system is two-dimensional.

We start from the variational expression for the electrical resistivity given by Ziman [14]:

$$
\rho=\frac{\frac{1}{k_{\mathrm{B}} T} \iiint\left(\phi_{\boldsymbol{k}}-\phi_{\boldsymbol{k}^{\prime}}\right)^{2} \mathcal{P}_{\boldsymbol{k}, \boldsymbol{q}}^{\boldsymbol{k}^{\prime}} \mathrm{d} \boldsymbol{k} \mathrm{d} \boldsymbol{k}^{\prime} \mathrm{d} \boldsymbol{q}}{\left|\frac{1}{2 \pi^{2}} \int e \boldsymbol{v}_{\boldsymbol{k}} \cdot \boldsymbol{u} \phi_{\boldsymbol{k}} \frac{\partial f_{\boldsymbol{k}}^{0}}{\partial \mathcal{E}_{\boldsymbol{k}}} \mathrm{d} \boldsymbol{k}\right|^{2}},
$$

where $\boldsymbol{k}, \boldsymbol{k}^{\prime}$ - electron wave vectors before and after scattering, respectively, $\boldsymbol{q}$ - phonon wave vector, $k_{\mathrm{B}}$ - the Boltzmann constant, $T$ - temperature, $\phi_{\boldsymbol{k}}$ - deviation 
from equilibrium of the electron distribution function, $\mathcal{P}_{\boldsymbol{k}, \boldsymbol{q}}^{\boldsymbol{k}^{\prime}}$ - transition probability from the state $\boldsymbol{k}$ to the state $\boldsymbol{k}^{\prime}$ due to electron-phonon interaction, $e-$ electron charge, $\boldsymbol{v}_{\boldsymbol{k}}$ - electron group velocity, $f_{k}^{0}$ - electron distribution function, $\varepsilon_{k}$ - electron energy.

We assume that the phonon system is in thermal equilibrium so that for phonon distribution function $\phi_{\boldsymbol{q}}=0$. Choice of the $\phi_{\boldsymbol{k}}$ function is not crucial and for our calculation we shall use the standard trial function

$$
\phi_{\boldsymbol{k}}=\boldsymbol{k} \cdot \boldsymbol{u},
$$

where $\boldsymbol{u}$ - unit vector in the direction of external field.

The denominator is then given by

$$
\left|\frac{1}{2 \pi^{2}} \int e \boldsymbol{v}_{\boldsymbol{k}} \cdot \boldsymbol{u} \phi_{\boldsymbol{k}} \frac{\partial f_{\boldsymbol{k}}^{0}}{\partial \mathcal{E}_{\boldsymbol{k}}} \mathrm{d} \boldsymbol{k}\right|^{2}=\left(\frac{e k_{\mathrm{F}} \varsigma}{4 \pi^{2} \hbar}\right)^{2},
$$

where $k_{\mathrm{F}}-$ Fermi radius, $\varsigma-$ Fermi circle circumference.

Much more effort must be made to calculate the numerator. Because calculations are complex we shall walk through them step by step. The transition probability due to electron-phonon interaction is

$$
\begin{aligned}
& \mathcal{P}_{\boldsymbol{k}, \boldsymbol{q}}^{\boldsymbol{k}^{\prime}}=\left(\frac{\pi}{m N_{\mathrm{A}} \omega}\right) \delta_{\boldsymbol{g}, \boldsymbol{k}^{\prime}-\boldsymbol{k}-\boldsymbol{q}}\left|\mathcal{F}_{\boldsymbol{q}, p}\left(\boldsymbol{k}, \boldsymbol{k}^{\prime}\right)\right|^{2} \\
& \quad \times \mathcal{O}\left(\varepsilon_{\boldsymbol{k}}+\hbar \omega-\varepsilon_{\boldsymbol{k}^{\prime}}\right) n_{\boldsymbol{q}}^{0} f_{\boldsymbol{k}}^{0}\left(1-f_{\boldsymbol{k}^{\prime}}^{0}\right),
\end{aligned}
$$

where $m$ - ion mass, $N_{\mathrm{A}}$ - number of unit cells per unit area, $n_{\boldsymbol{q}}^{0}$ - phonon occupation number, $\boldsymbol{g}$ - reciprocal lattice vector, $p$ - polarization of the phonon, $\hbar \omega$ is the phonon energy and $\mathcal{O}$ is the Landau symbol.

For longitudinal phonons we find the $\mathcal{F}_{q, p}\left(\boldsymbol{k}, \boldsymbol{k}^{\prime}\right)$ function to be

$$
\mathcal{F}_{\boldsymbol{q}, p}\left(\boldsymbol{k}, \boldsymbol{k}^{\prime}\right)=\boldsymbol{e}_{\boldsymbol{q}, p} \cdot\left(\boldsymbol{k}^{\prime}-\boldsymbol{k}\right) \cdot \ell\left(\left|\boldsymbol{k}^{\prime}-\boldsymbol{k}\right|\right),
$$

where $e_{\boldsymbol{q}, p}$ is the polarization vector of the phonon and $\ell(K)$ is a quantity which has the dimension of energy and depends on the magnitude of the scattering vector

$$
\boldsymbol{K}=\boldsymbol{k}^{\prime}-\boldsymbol{k} \text {. }
$$

Putting all this together we can write down the variational integral

$$
\begin{aligned}
& \frac{\pi}{k_{\mathrm{B}} T m N_{\mathrm{A}} \omega} \iiint(\boldsymbol{K} \cdot \boldsymbol{u})^{2}(\boldsymbol{K} \cdot \boldsymbol{e})^{2} \ell^{2}(K) f_{\boldsymbol{k}}^{0}\left(1-f_{\boldsymbol{k}^{\prime}}^{0}\right) \\
& \times n_{\boldsymbol{q}}^{0} \mathcal{O}\left(\varepsilon_{\boldsymbol{k}}+\hbar \omega-\varepsilon_{\boldsymbol{k}^{\prime}}\right) \delta_{\boldsymbol{g}, \boldsymbol{k}^{\prime}-\boldsymbol{k}-\boldsymbol{q}} \mathrm{d} \boldsymbol{k} \mathrm{d} \boldsymbol{k}^{\prime} \mathrm{d} \boldsymbol{q} .
\end{aligned}
$$

Using the same simplifying method as Ziman [14] we obtain the following equation for electrical resistivity:

$$
\begin{aligned}
\rho= & \frac{2 \pi \hbar}{e^{2} m N_{\mathrm{A}} k_{\mathrm{B}} T} \frac{1}{k_{\mathrm{F}}^{2} \varsigma^{2}} \\
& \times \iint \frac{(\boldsymbol{K} \cdot \boldsymbol{u})^{2}(\boldsymbol{K} \cdot \boldsymbol{e})^{2} \ell^{2}(K)}{\left(1-\mathrm{e}^{-\hbar \omega / k_{\mathrm{B}} T}\right)\left(\mathrm{e}^{\hbar \omega / k_{\mathrm{B}} T}-1\right)} \frac{\mathrm{d} \varsigma}{v} \frac{\mathrm{d} \varsigma^{\prime}}{v^{\prime}},
\end{aligned}
$$

where $v$ is the electron velocity.

Following Ziman [14] we assume that $N$-processes are the only scattering processes taking place. For this processes the polarisation vector $\boldsymbol{e}$ is parallel to $\boldsymbol{q}$, which then equals $\boldsymbol{K}$. We can write that

$$
(\boldsymbol{K} \cdot \boldsymbol{e})^{2}=K^{2}=q^{2},
$$

$$
(\boldsymbol{K} \cdot \boldsymbol{u})^{2}=\frac{1}{2} K^{2}=\frac{1}{2} q^{2} .
$$

We calculate the resistivity $\rho$ given by Eq. (8) changing the variables in the first integral over $\mathrm{d} \varsigma$ to $k_{\mathrm{F}} \mathrm{d} \phi$ and integrating over $\mathrm{d} \varsigma^{\prime}$ which is immediate and equals $\varsigma$. The angle $\phi$ is between $\boldsymbol{k}$ and $\boldsymbol{k}^{\prime}$ vectors. Then we obtain

$$
\begin{aligned}
\rho= & \frac{\pi \hbar}{e^{2} m N_{\mathrm{A}} k_{\mathrm{B}} T} \frac{1}{k_{\mathrm{F}}^{2} v_{\mathrm{F}}^{2}} \frac{1}{\varsigma} \\
& \times \int \frac{q^{4} \ell^{2}(q) k_{\mathrm{F}} \mathrm{d} \phi}{\left(1-\mathrm{e}^{-\hbar \omega / k_{\mathrm{B}} T}\right)\left(\mathrm{e}^{\hbar \omega / k_{\mathrm{B}} T}-1\right)} .
\end{aligned}
$$

To accomplish the calculations, all we need is to find $k_{\mathrm{F}} \mathrm{d} \phi$ expressed by the scattering vector $q$. This can be done by using the law of cosines in the form

$$
\begin{aligned}
& q^{2}=k^{\prime 2}+k^{2}-2 k^{\prime} k \cos \phi \Longrightarrow q \mathrm{~d} q=k_{\mathrm{F}}^{2} \sin \phi \mathrm{d} \phi, \\
& q^{2}=k^{\prime 2}+k^{2}-2 k^{\prime} k \cos \phi \Longrightarrow \cos \phi=1-\frac{q^{2}}{2 k_{\mathrm{F}}^{2}} .
\end{aligned}
$$

Finally the equation describing the resistivity of an ideal $2 \mathrm{D}$ metallic system is

$$
\begin{aligned}
& \rho_{2 \mathrm{D}}=\frac{\hbar}{2 e^{2} m N_{\mathrm{A}} k_{\mathrm{B}} T k_{\mathrm{F}}^{4} v_{\mathrm{F}}^{2}} \\
& \times \int_{0}^{D} \frac{q^{5} \ell^{2}(q) \mathrm{d} q}{\sqrt{1-\left(1-\frac{q^{2}}{2 k_{\mathrm{F}}^{2}}\right)^{2}}\left(1-\mathrm{e}^{-\hbar \omega / k_{\mathrm{B}} T}\right)\left(\mathrm{e}^{\hbar \omega / k_{\mathrm{B}} T}-1\right)},
\end{aligned}
$$

where $D$ is the Debye radius in two dimensions, $\ell(q)=$ $n / N\left(\varepsilon_{\mathrm{F}}\right)$ where $N\left(\varepsilon_{\mathrm{F}}\right)$ - density of electron levels at the Fermi surface and $\omega=\omega(q)$. For comparison, below we write the Bloch-Grüneisen formula for the electrical resistivity which we mentioned in the introduction

$$
\begin{aligned}
& \rho_{3 \mathrm{D}}=\frac{3 \pi \hbar}{4 e^{2} m N k_{\mathrm{B}} T k_{\mathrm{F}}^{4} v_{\mathrm{F}}^{2}} \\
& \times \int_{0}^{D} \frac{q^{5} \ell^{2}(q) \mathrm{d} q}{\left(1-\mathrm{e}^{-\hbar \omega / k_{\mathrm{B}} T}\right)\left(\mathrm{e}^{\hbar \omega / k_{\mathrm{B}} T}-1\right)} .
\end{aligned}
$$

There is another matter which should be thoroughly considered. The real phonon dispersion relation, $\omega(q)$, is not linear and the actual form of this dispersion relation is

$$
\omega(q)=\omega_{0} \sin \left(\frac{q a}{2}\right),
$$

where $\omega_{0}-$ characteristic frequency, $a$ - the lattice constant.

However, Eq. (15) gives too large values of the sound velocity at the Brillouin zone boundaries. Therefore we do not use the real dispersion relation but we replace it with the one for which the velocity of sound $s$ is expressed by the formula

$$
s=\frac{k_{\mathrm{B}} \theta}{\hbar D},
$$

where $\theta$ is the Debye temperature. This formula gives more realistic values for $s$ and suggests the following dispersion relation for phonons:

$$
\omega(q)=\frac{k_{\mathrm{B}} \theta}{\hbar D} q .
$$




\section{Results}

We carried out the calculations of the resistivity in the range of temperature from $T=0.04 \theta$ to $T=1.4 \theta$ (the numerical results of the investigated quantities did not significantly change for higher temperatures). We calculated the ratio of the $2 \mathrm{D}$ resistivity $\rho_{2 \mathrm{D}}$ to the $3 \mathrm{D}$ resistivity $\rho_{3 \mathrm{D}}$, given by the Bloch-Grüneisen formula in order to show the influence of dimensionality on the resistivity. We used the linear dispersion relation in both cases. The result is shown in Fig. 1.

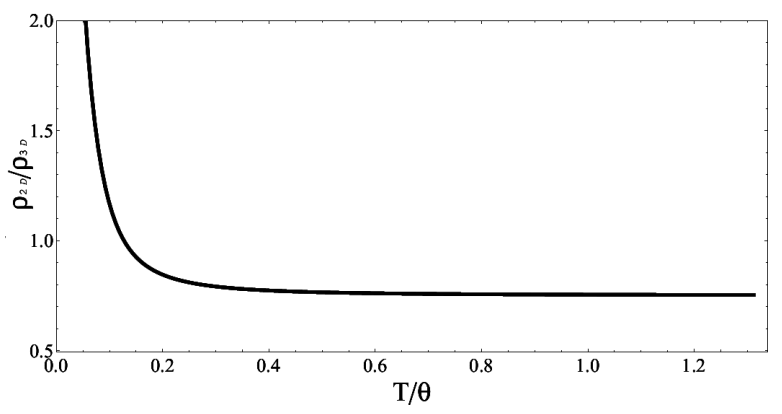

Fig. 1. The relative resistivity $\rho_{2 \mathrm{D}} / \rho_{3 \mathrm{D}}$ (symbols explained in the text) vs. reduced temperature $T / \theta$.

To make the ratio dimensionless we need to multiply $2 \mathrm{D}$ resistivity by the lattice constant $a$. In a wide range of temperatures the resistivity of a monolayer is about 0.75 of that of the bulk sample. It can be explained in a simple way: the charge carriers in $2 \mathrm{D}$ space have less final states to scatter thus the current is less damped. However, below $T=0.2 \theta$ we obtained the unexpected result: the $2 \mathrm{D}$ resistivity rises up and becomes greater than 3D one below $0.1 \theta$. It can be explained in this way: for very low temperatures the exponent $\hbar \omega / k_{\mathrm{B}} T$ is large and we can approximate the part of the function under the integral in (13) and (14) which is dependent on temperature by $\exp \left(-\hbar \omega / k_{\mathrm{B}} T\right)$. On the other hand, this factor is equal to $\exp (-\theta q / T D)$. It resembles the well-known fact that at low temperatures the phonons of small $q$ 's give main contribution to electron scattering. This contribution is essentially greater in our $2 \mathrm{D}$ model because of the square root expression in the denominator which tends to zero.

\section{Conclusions}

We have investigated the influence of temperature on the electrical resistivity of a $2 \mathrm{D}$ metallic layer. The electrical resistivity of such system is smaller than the resistivity of a bulk material in a great range of temperatures and exceeds the bulk resistivity for low temperatures. Both effects can be explained by the fact of low dimensionality of the considered sample. Our model is strictly two-dimensional and is difficult for experimental verification however it can be used for a semi-quantitative analysis of the samples strongly reduced in one dimension. Therefore our next goal is to investigate how the resistivity changes with temperature for film thickness greater than one atomic layer.

\section{Acknowledgments}

This work has been partly supported by the EU Human Capital Operation Program, Polish Project No. POKL.04.0101-00-434/08-00. It was also partially supported by the Polish Ministry of Science and Higher Education and its grants for Scientific Research.

\section{References}

[1] K. Fuchs, Math. Proc. Cambridge 34, 100 (1938).

[2] E.H. Sondheimer, Adv. Phys. 1, 1 (1952).

[3] V.B. Sandomirskii, J. Exp. Theor. Phys. 52, 158 (1967).

[4] N.W. Ashcroft, N. Trivedi, Phys. Rev. B 38, 12298 (1988).

[5] G. Palasantzas, J.Th.M. De Hosson, Phys. Rev. B 63, 125404 (2001).

[6] A.E. Meyerovich, I.V. Ponomarev, Phys. Rev. B 65 155413 (2002).

[7] D.V. Fedorov, P. Zahn, I. Mertig, Thin Solid Films 515, 6921 (2007).

[8] A.E. Yarimbiyik, H.A. Schafft, R.A. Allen, M.D. Vaudin, M.E. Zaghloul, Microelectron Reliabil. 49, 127 (2009).

[9] S.V. Kravchenko, T.M. Klapwijk, Phys. Rev. Lett. 84, 2909 (2000).

[10] F. Bloch, Z. Phys. 52, 555 (1929).

[11] F. Bloch, Z. Phys. 59, 208 (1930).

[12] D.K. Ferry, S.M. Goodnick, J. Bird, Transport in Nanostructures, Cambridge University Press, Cambridge 2009.

[13] H.E. Camblong, P.M. Levy, Phys. Rev. Lett. 69 , 2835 (1992).

[14] J.M. Ziman, Electrons and Phonons, Clarendon Press, Oxford 1960. 